\title{
The origin and evolution of magnetic fields in clusters of galaxies
}

\author{
Luigina Feretti \\ INAF Istituto di Radioastronomia \\ Via P. Gobetti n. 101, 40129 Bologna, Italy \\ E-mail: Iferetti@ira.inaf.it
}

The origin and evolution of magnetic fields in the Universe is one of the most fascinating and currently unsolved problems in astrophysics. The existence of kpc-scale fields, in particular, raises several problems because of the need of amplification mechanisms acting with large efficiency on large scales. Clusters of galaxies, being the largest systems in the Universe, represent an ideal laboratory to test theories for the origin of extragalactic magnetic fields. Magnetic fields are investigated in the radio band from studies of the diffuse synchrotron emission and from the rotation measure of polarized radio sources. Observations show that cluster fields are at the $\mu \mathrm{G}$ level, with values up to tens of $\mu \mathrm{G}$ at the center of cooling core clusters. The origin of these magnetic fields is still debated. The SKA will deliver new data to address currently unaswered questions about magnetic fields in clusters and map the origin and evolution of magnetized structures in the Universe.

First MCCT-SKADS Training School

September 23-29, 2007

Medicina, Bologna Italy 


\section{Introduction}

Observations indicate that most of the Universe is permeated by magnetic fields. Celestial objects are magnetized and magnetic fields of significant strength are found everywhere over small and very large scales, in the interstellar space and in the extragalactic universe. In general, small compact objects have the largest magnetic field strengths, while bigger low-density objects have weaker magnetic fields.

Values of magnetic fields are in a wide range, spanning from the $\sim 10^{12} \mathrm{G}$ in pulsars and neutron stars to the $\sim 10^{3}$ G fields in stars, $\sim$ G fields in planets, $\sim 1 \mathrm{mG}$ in filaments of the Galaxy, $\sim 10 \mu \mathrm{G}$ in the spiral galaxies, and $\sim \mu \mathrm{G}$ in the radio emitting lobes of radio galaxies. Fields of intensity of the order of $\sim \mu \mathrm{G}$, or weaker, are detected in the intracluster space of clusters of galaxies, and in more rarefied regions of the intergalactic space. Upper limits of $\simeq 10^{-8}-10^{-9} \mathrm{G}$ have been obtained for the cosmological fields at large redshift.

The magnetic fields that we observe in the local Universe probably owe their present strength to dynamo amplification. In many astrophysical contexts, the dynamical scales are very short, thus even a very small primordial magnetic field may be largely amplified through a huge number of e-foldings. The magnetic fields on large-scale, instead, pose a more challenging problem, because the dynamical scales for large-scale objects are long, and thus the amplification is correspondingly slow. This implies the need for seed fields of non negligible intensity; on the contrary, there are good reasons to expect very small fields in the early Universe (e.g. [42], and references therein). In this framework, it is very important to derive firm values of the large-scale magnetic fields in clusters and in the intergalactic space, in order to constrain the values of seed fields that may have been at their origin and thus constrain the evolution of magnetic fields in the Universe. Clusters of galaxies, being the largest systems in the Universe, represent an ideal laboratory to test theories for the origin of extragalactic magnetic fields.

The investigation of cosmic magnetic fields and of their origin and evolution has been identified as one of the five Key Science Projects for the Square Kilometer Array (SKA) [26]. Indeed, it has been recognized that this study can address unanswered questions in fundamental (astro)physics, is science which is unique to the radio band and to the SKA, excites the broader community and is of interest to funding agencies. Moreover, SKA will certainly discover new magnetic phenomena, that are presently unknown.

In this paper the current knowledge on magnetic field in clusters is reviewd, and the contribution that the SKA will give to the knowledge of magnetic fields in the Universe is illustrated. The intrinsic parameters quoted in this paper are computed with a Hubble constant $\mathrm{H}_{0}=70 \mathrm{~km} \mathrm{~s}^{-1}$ $\mathrm{Mpc}^{-1}$ and a deceleration parameter $\mathrm{q}_{0}=0.5$.

\section{Cluster magnetic fields}

The study of cluster magnetic fields has gained a big interest in recent years, leading to several new observations as well as simulations. There are, however, still many questions to answer: does the field strength depend on cluster parameters such as the gas temperature, metallicity, mass, substructure and density profile, how do the fields extend, what is the radial trend of the field 


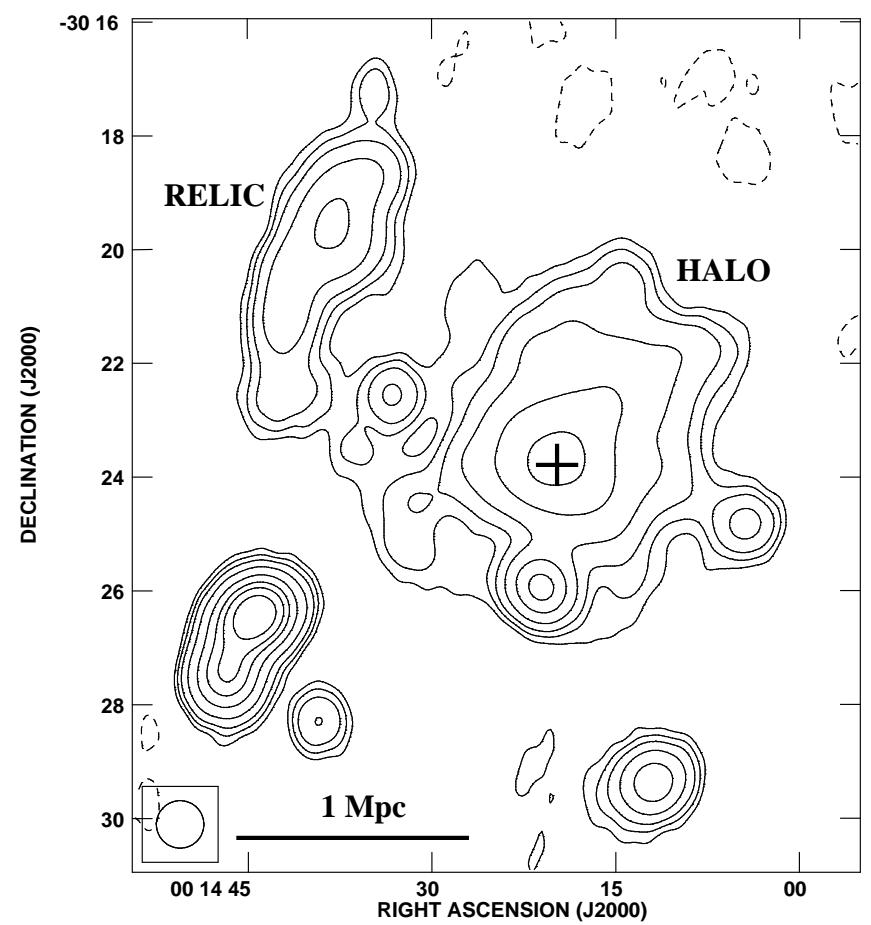

Figure 1: Radio emission of the cluster A $2744(\mathrm{z}=0.308)$ at $1.4 \mathrm{GHz}$. The cluster center is indicated by a cross. The image shows the two diffuse radio sources: a central giant radio halo, and a peripheral elongated relic ([29, ,41]).

strength, what are the coherence scales, how do the fields evolve with cosmic time, and finally how were the fields generated?

\subsection{Synchrotron diffuse emission}

The presence of magnetic fields in clusters is directly demonstrated by the existence of largescale diffuse radio sources which are related to the intracluster medium (ICM) rather than to individual radio galaxies. These sources are known as radio halos and relics. The radio emission is of synchrotron origin, thus its total intensity is related to the strength of the magnetic field, while the polarized intensity gives information about i) the orientation of the magnetic field in the plane of the sky (from the polarization position angle) and ii) the field degree of ordering (from the polarization percentage). Detailed formulae and their derivations are given in Govoni \& Feretti [31] and Feretti \& Giovannini [24].

The Coma cluster is the first cluster where a radio halo (Coma $\mathrm{C}$ [50], [27]) and a relic $(1253+275$ [ $₫$ ) have been detected. The radio halos are permeating the cluster central regions, with a brightness distribution similar to that of the X-ray gas and a typical extent of $\gtrsim 1 \mathrm{Mpc}$ (see e.g. the radio halo in the cluster A 2744 shown in Fig. 1). Halos are characterized by steep radio spectrum. Limits of a few percent have been derived for their polarized emission (except for the filaments in A 2255 [32]). Relic sources are similar to halos in their low surface brightness, large 
size and steep spectrum, but they are typically found in cluster peripheral region. Several clusters showing a halo do also host a relic (e.g. A 2744 in Fig. 1). Unlike halos, relics are highly polarized $(\sim 20-30 \%)$.

From radio data, it is derived that under equipartition conditions magnetic field strengths in halos and relics range from $\simeq 0.1$ to $1 \mu \mathrm{G}$. These calculations typically assume equal energy in relativistic protons and electrons $(\mathrm{k}=1)$, a volume filling factor $\Phi=1$, a low frequency cut-off of $10 \mathrm{MHz}$, and a high frequency cut-off of $10 \mathrm{GHz}$. In A 2256, high fractional polarization in the relic indicates large regions $(500 \mathrm{kpc})$ of fairly uniform magnetic field direction [13]. Recent reviews on diffuse cluster radio sources are presented by Giovannini \& Feretti [28], Feretti [23] and Feretti \& Giovannini [24].

\subsection{Rotation measure}

An independent way of obtaining information about the cluster magnetic field, using data at radio wavelengths, is represented by the analysis of the Faraday rotation of radio sources in the background of clusters or in the galaxy clusters themselves. With this method, shortly illustated below, an indirect measurement of the strength and structure of the field along the line of sight is obtained.

The synchrotron radiation from cosmic radio sources is well known to be linearly polarized. A linearly polarized wave of wavelength $\lambda$, traveling from a radio source through a magnetized medium, experiences a phase shift of the left versus right circularly polarized components of the wavefront, leading to a rotation $\Delta \chi$ of the position angle of the polarization, according to the law: $\Delta \chi=\mathrm{RM} \lambda^{2}$, where RM is the Faraday rotation measure. The RM is related to the electron density $n_{\mathrm{e}}$, in $\mathrm{cm}^{-3}$, and to the magnetic field along the line of sight $B_{\|}$, in $\mu \mathrm{G}$, through the relation:

$$
\mathrm{RM}=812 \int_{0}^{L} n_{\mathrm{e}} B_{\|} d l \quad \text { rad } m^{-2},
$$

where the path length $l$ is in kpc. By convention, RM is positive (negative) for a magnetic field directed toward (away from) the observer.

The RM values can be derived from multifrequency polarimetric observations of sources within or behind the clusters, by measuring the position angle $\chi$ of the polarized radiation as a function of wavelength. In general, the position angle must be measured at three or more wavelengths in order to determine RM accurately and remove the position angle ambiguity: $\chi_{\text {true }}=\chi_{\text {obs }} \pm n \pi$. Once the contribution of our Galaxy is subtracted, the RM should be dominated by the contribution of the ICM, and therefore it can be combinedth measurements of $n_{e}$ to estimate the cluster magnetic field along the line of sight.

In simple cases, i.e. if the density and magnetic field strenght of the Faraday screen can be approximated by simple geometries, it is possible to derive analytical solutions of the integral 2.1. thus infer the strength of the magnetic field from RM measurements. In more complex and realistic cases, the solution of the integral is not possible analytically and numerical methods or a semianalytical approach are needed ([40], [18]). A recent tecnique to analyse and interpret the RM data is the RM Synthesis, developed by Brentjens \& De Bruyn [8], which uses the RM transfer 


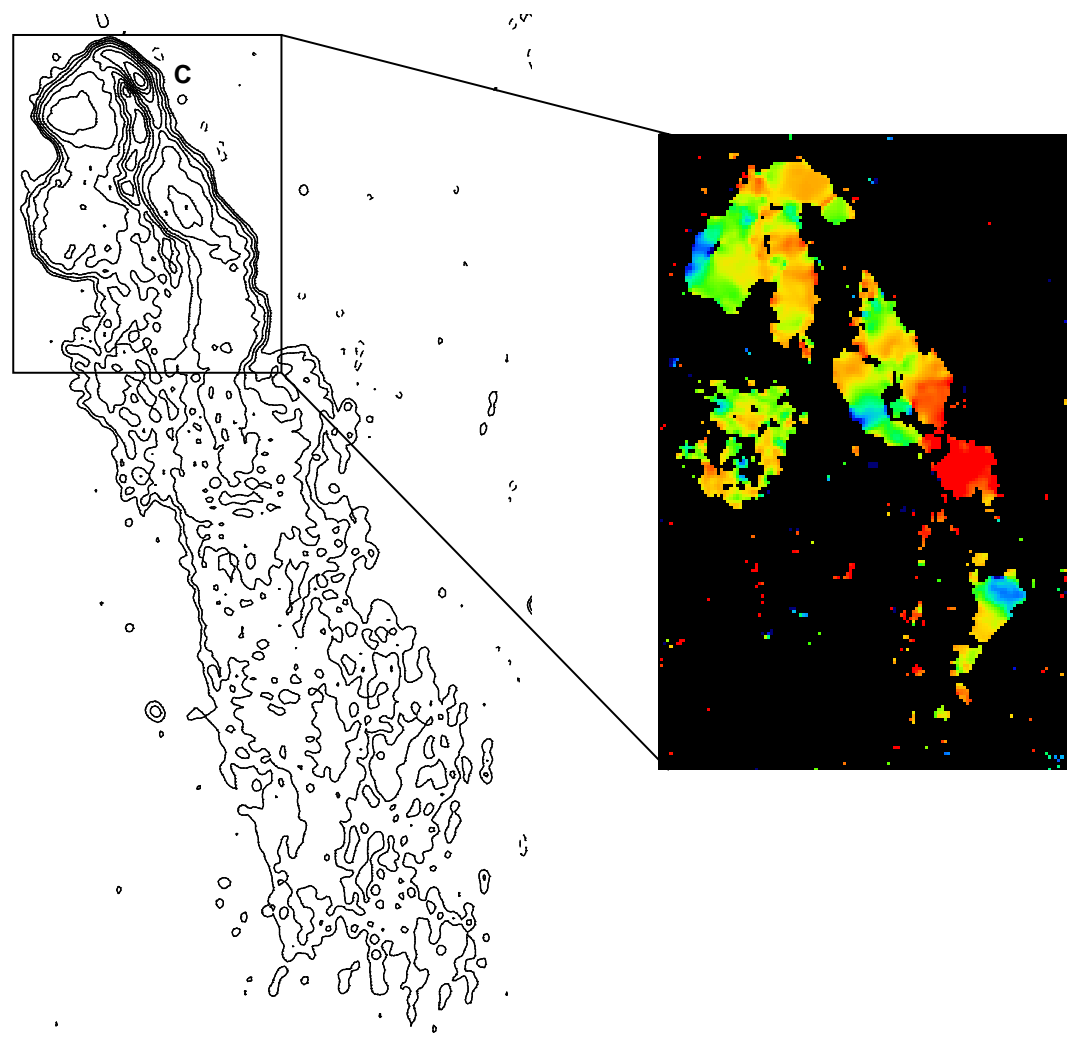

Figure 2: VLA contour plot of the tailed radio galaxy 0053-015 in A 119 at $1.4 \mathrm{GHz}$ (left), and RM image (right). The values of RM range between $-350 \mathrm{rad} \mathrm{m}^{-2}$ and $+450 \mathrm{rad} \mathrm{m}^{-2}$, with $<\mathrm{RM}>=+28 \mathrm{rad} \mathrm{m}^{-2}$, and a dispersion of $\sigma_{R M}=152 \mathrm{rad} \mathrm{m}^{-2}$. They show fluctuations on scales of $\sim 3.5 \operatorname{arcsec}[20]$.

function to solve the $n \pi$ ambiguity related to the RM computation, and allows to distinguish the emission as a function of Faraday depth.

\subsection{Current results from RM studies}

Cluster surveys of the Faraday rotation measures of polarized radio sources both within and behind clusters provide an important probe of the existence of intracluster magnetic fields. The observing strategy to derive information on the magnetic field intensity and structure is twofold: i) obtain the average value of the RM of sources located at different impact parameters of the cluster, ii) derive maps of the RM of extended radio sources, to evaluate the $\sigma$ of the RM distribution. The RM values derived in background or embedded cluster sources are of the order of tens to thousands $\operatorname{rad~}^{-2}$ (Fig. 2).

Studies have been carried out on both statistical samples and individual clusters (see e.g. the review by Govoni \& Feretti [31] and references therein). From statistical studies, Clarke et al. [11] analyzing RMs for a representative sample of 16 cluster sources, plus a control sample, found a statistically significant broadening of the RM distribution in the cluster sample, and a clear increase in the width of the RM distribution toward smaller impact parameter (see Fig. 3). They derived that the ICM is permeated with a high filling factor by magnetic fields at levels of $4-8 \mu \mathrm{G}$ and with a 


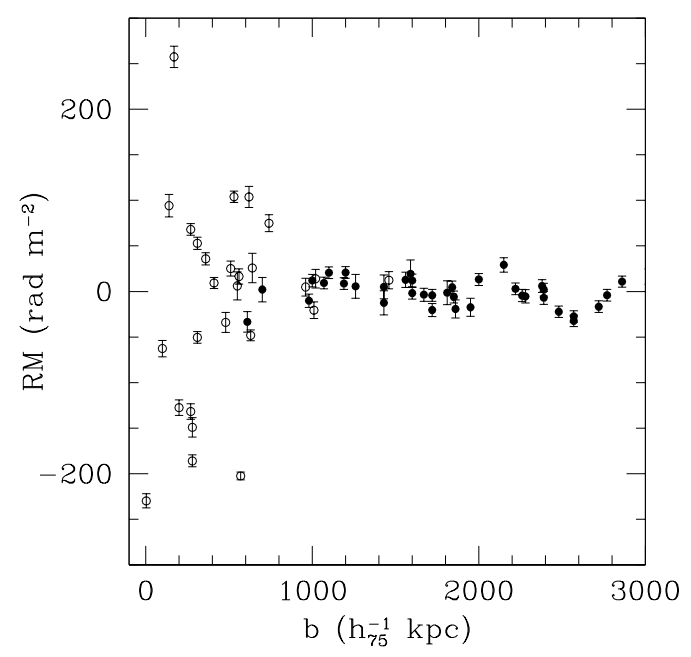

Figure 3: Galaxy-corrected rotation measure plotted as a function of source impact parameter in kiloparsecs for the sample of sources from Clarke et al. [11]. Open dots refer to cluster sources, closed dots to control sources.

correlation length of $\sim 15 \mathrm{kpc}$, up to $\sim 0.75 \mathrm{Mpc}$ from the cluster center. The results are confirmed by new data on an expanded sample [12].

The first detailed studies of RM on individual clusters have been performed on cooling core clusters, owing to the extremely high RMs of the powerful radio galaxies at their centers (e.g., Hydra A [46], 3C295 [1]). High values of the magnetic fields, up to tens of $\mu \mathrm{G}$, have been obtained, but they only refer to the innermost cluster regions. Studies on larger are as of clusters have been carried out e.g. for Coma [19], A 119 [20], A 514 ([30], 3C 129 [47], A 2255 [33].

Overall, the data are consistent with cluster atmospheres containing magnetic fields in the range of 1-5 $\mu \mathrm{G}$, regardless of the presence or not of diffuse radio emission. At the center of cooling core clusters, magnetic field strenghts can be larger by more than a factor of 2 . The RM distributions are generally patchy, indicating that large-scale magnetic fields are not regularly ordered on cluster scales, but have coherence scales between 1 and $50 \mathrm{kpc}$ (see also next subsection). In most clusters the fields are not dynamically important, with magnetic pressures much lower than the thermal pressures, but the fields may play a fundamental role in the suppression of the particle thermal conduction [10] and in the energy budget of the ICM.

\subsection{Magnetic field structure}

The simplest model is a uniform field throughout the cluster. However, this is not realistic: if the field values detected at the cluster centers would extend over several core radii, up to distances of the order of $\sim \mathrm{Mpc}$, the magnetic pressure would exceed the thermal pressure in the outer parts of the clusters. The magnetic field intensity is likely to decrease with the distance from the cluster center, as derived in Coma [9]. This is also predicted as a result of compression of the thermal plasma during the cluster gravitational collapse, where the magnetic field lines are frozen into the plasma, and compression of the plasma results in compression of flux lines. As a consequence of 
magnetic flux conservation, the expected growth of the magnetic field is proportional to the gas density as $\mathrm{B} \propto \rho^{2 / 3}$.

Dolag et al. [16] showed that in the framework of hierarchical cluster formation, the correlation between two observable parameters, the RM and the X-ray surface brightness, is expected to reflect the correlation between the magnetic field and gas density. Therefore, from the analysis of the RM versus X-ray brightness it is possible to infer the trend of magnetic field versus gas density. The application of this approach to A 119 has given a radial profile of the magnetic field scaling with the gas density as $B \propto n_{e}^{0.9}[16]$. The magnetic field decline with radius is confirmed in this case.

Another important aspect to consider is the structure in the cluster magnetic field, i.e. the existence of filaments and flux ropes [17]. The magnetic field structure can be investigated by deriving the power spectrum of the field fluctuations, defined as: $\left|B_{\kappa}\right|^{2} \propto \kappa^{-n}$, where $\kappa$ represents the wave number of the fluctuation scale. By using a semi-analytic technique, Enßlin \& Vogt [18] and Vogt \& Enßlin [48] showed that the magnetic field power spectrum can be estimated by Fourier transforming RM maps if very detailed RM images are available. Alternatively, a numerical approach using Monte Carlo simulations has been developed by Murgia et al. [40] to reproduce the rotation measure and depolarization produced by magnetic field with different power spectra.

\subsection{Origin of intracluster magnetic fields}

The field strengths that we observe in clusters greatly exceed the amplitude of the seed fields produced in the early universe, or fields injected by some mechanism by high redshift objects [42]. There are two basic possibilities for their origin: 1) ejection from galactic winds of normal galaxies or from active and starburst galaxies ([38], [49]);2) amplification of seed fields during the cluster formation process.

Support for a galactic injection in the ICM comes from the evidence that a large fraction of the ICM is of galactic origin, since it contains a significant concentration of metals. However, fields in clusters have strengths and coherence size comparable to, and in some cases larger than, galactic fields [34]. Therefore, it seems quite difficult that the magnetic fields in the ICM derive purely from ejection of the galactic fields, without invoking other amplification mechanisms ([14], [43]).

Magnetic field amplication is likely to occur during the cluster collapse, simply by compression of an intergalactic field. Clusters have present day overdensities $\rho \sim 10^{3}$ : in order to get $B_{\mathrm{ICM}}>10^{-6} \mathrm{G}$ by adiabatic compression $\left(B \propto \rho^{2 / 3}\right)$ requires intergalactic (seed) fields of at least $10^{-8} \mathrm{G}$. These are somewhat higher than current limits derived in the literature ([5], [7]). A possibility to obtain a larger field amplification is through cluster mergers. Mergers generate shocks, bulk flows and turbulence within the ICM. The first two of these processes can result in some field amplification simply through compression. However, it is the turbulence which is the most promising source of non-linear amplification.

MHD calculations have been performed ([15], [44], [45]) to investigate the evolution of magnetic fields. The results of these simulations show that cluster mergers can dramatically alter the local strength and structure of cluster-wide magnetic fields, with a strong amplification of the magnetic field intensity. Shear flows are extremely important for the amplification of the magnetic field, while the compression of the gas is of minor importance. The initial field distribution at the beginning of the simulations at high redshift is irrelevant for the final structure of the magnetic field. The final structure is dominated only by the cluster collapse. Fields can be amplified from seed 
fields of $\sim 10^{-9} \mathrm{G}$ at $\mathrm{z}=15$ to $\sim 10^{-6} \mathrm{G}$ at the present epoch [15]. Roettiger et al. [44] found a significant evolution of the structure and strength of the magnetic fields during two distinct epochs of the merger evolution. In the first, the field becomes quite filamentary as a result of stretching and compression caused by shocks and bulk flows during infall, but only minimal amplification occurs. In the second, amplification of the field occurs more rapidly, particularly in localized regions, as the bulk flow is replaced by turbulent motions. In conclusion, mergers change the local magnetic field strength drastically, but also the structure of the cluster-wide field is influenced. At early stages of the merger the filamentary structures prevail. This structure breaks down later $(\sim$ 2-3 Gyr) and leaves a stochastically ordered magnetic field. Subramanian et al. [45] argue that the dynamo action of turbulent motions in the intracluster gas can amplify a random magnetic field by a net factor of $10^{4}$ in 5 Gyr. The field is amplified by random shear, and has an intermittent spatial distribution, making possibly filaments.

\section{Magnetic fields in the intergalactic medium}

Recent attempts to detect diffuse radio emission beyond clusters, i.e. in very rarefied regions of the intergalactic space, have shown recent promise in imaging diffuse synchrotron radiation of very low level. A very faint emission has been detected at $327 \mathrm{MHz}$ with the WSRT in the Coma cluster region between the radio halo Coma $\mathrm{C}$ and the relic 1253+275 (e.g. [36], [39]). The equipartition magnetic field in this region is $\simeq 10^{-7} \mathrm{G}$.

Another possible evidence of radio emission in the intergalactic medium is found in the filament of galaxies $\mathrm{ZwCl} 2341.1+0000$ at $\mathrm{z} \sim 0.3$ [2]]. The radio emission spans over several Mpc following the filamentary network of galaxies as traced by optical redshift surveys. Recent results that provide further evidence for intergalactic magnetic fields have been presented by Kronberg [39]. In addition, the giant ring-like features detected in A 3376 [3] have been suggested to be related to the large-scale structure formation process, rather than to the cluster. All these data support the existence of an intergalactic magnetic field more widespread and somewhat lower than that in the intracluster medium within clusters. It could be possibly associated with large-scale shocks related to the formation of the large-scale structure in the universe, as modeled by Keshet et al. [35]. This field may represent the seed field for galaxies and clusters, and may play an important role in the formation of large scale structure.

\section{Magnetic field studies with the Square Kilometer Array}

The SKA will deliver new data that will address the currently unanswered questions about the cosmic magnetism (see [25] and [6] for details). Owing to its high sensitivity, in a one-hour observation of a $0.1 \mathrm{mJy}$ source with a fractional linear polarization of $1 \%$, the SKA will make a $10 \sigma$ detection of linear polarization, resulting in an error of about $5 \mathrm{rad} \mathrm{m}^{-2}$ in $\mathrm{RM}$ and an error of $\sim 10^{\circ}$ in intrinsic polarization angle.

The main project for the study of cosmic magnetism is a wide-field SKA survey for RMs, at $1.4 \mathrm{GHz}$ continuum and polarization, down to a rms sensitivity of $0.1 \mu \mathrm{Jy}$. This will provide RMs for about $310^{7}$ compact polarized extragalactic sources over the entire sky, spaced by $\sim 90^{\prime \prime}$, expanding the sample of RM measurements by five orders of magnitude over current data sets. 
Since RM values are produced by Faraday rotation in the foreground magneto-ionic structures, the RM grid will allow the development of three-dimensional models of the magnetized gas in i) the Galaxy, ii) the external galaxies, iii) the galaxy clusters, thus providing detailed information on the magnetic fields in these systems.

Within the Galaxy, RMs will be obtained for $\sim 20000$ pulsars. Their dispersion measures will be combined with the RM values to yield the Galactic magnetic field. It will be possible to resolve small scale features, in particular those due to turbulence and other local effects. Data on external galaxies will provide information on magnetic fields up to large galactic radii, for a wide range of inclinations, galaxy types and environments, and search for magnetic reconnection regions which may heat the gas and accelerate particles.

In clusters, such a data-base, when combined with redshift and X-ray information from future instruments, will allow a statistical analysis, leading to a great improvement over the current studies, which are limited to the few strongest sources in each cluster (see also [21]). With the all sky RM survey, it will be possible to obtain about 500 RM measurements through lines of sight of nearby clusters, and at least $10-20 \mathrm{RMs}$ through clusters at redshifts $\gtrsim 0.5$. This will give not only the strength of the magnetic field, but also its structure, profile, coherence length (power spectrum), and filling factor.

In addition to the RM survey, deep multifrequency observations, targeting individual clusters, will allow the investigation of the intensity and structure of the ICM magnetic fields and analyse the correlation between the magnetic field intensity and the gas density, get information on the magnetic field profile and test models of magnetic field formation. Moreover, low surface brightness radio features such as relics and halos should be detected in their thousands [22] allowing us to explore the role of dynamically important magnetic fields in merging clusters and providing vital clues to the origin of cluster magnetic fields.

Finally, using the described techniques, magnetic fields can also be observed and studied in the jets and lobes of radio galaxies. The largely improved sensitivity and resolution of SKA will allow the study of faint objects and of the low brightness components of extended radio sources, the distinction of local features, the discrimination between internal and external Faraday dispersion, the connection between magnetic fields within the radio galaxies and the cosmological magnetic fields.

\subsection{Intergalactic medium and cosmic web}

The detection of synchrotron radiation at the lowest possible levels will allow the measurement of magnetic fields in even more rarefied regions of the intergalactic space, and the investigation of the relation between the formation of magnetic fields and the formation of the large-scale structure in the universe.

The high density peaks of the cosmic filaments produced by the gravitational infall can be detected by mapping the diffuse synchrotron emission arising from a combination of particle acceleration and the extended cosmic magnetic field. SKA observations can therefore be used to trace the extended baryon component of the cosmic web. Fields of $B \simeq 10^{-9}-10^{-8} \mathrm{G}$ are expected along filaments of $10 \mathrm{Mpc}$ length with $n_{e} \simeq 10^{-5} \mathrm{~cm}^{-3}$ electron density [39] which yield Faraday rotation measures of $\mathrm{RM}=0.1-1 \mathrm{rad} \mathrm{m}^{-2}$. To measure such low RMs needs high angular resolution and accurate subtraction of the Galactic foreground. 
The study of the RM of distant quasars could provide an independent information on the magnetic field in the intergalactic medium [39]. In addition, the angular correlation function of RMs for a given redshift can be used to deduce the primordial magnetic field power spectrum [37].

\subsection{High redshift objects}

Dramatic improvements are expected in the study of magnetic fields at high redshift, both in galaxies and protogalaxies. SKA will identify RMs towards millions of quasars. In particular, most quasars are known to have foreground clouds along the line of sight, as demonstrated by the Lyman- $\alpha$ absorbers at $\mathrm{z} \sim 1-3$. If both the RM and the redshift of a quasar is known, it will be possible to trace the evolution of RM with redshift. Moreover, SKA will identify many polarized sources at $\mathrm{z}>3$, and possibly obtain RM for them. This will prove the strength of magnetic fields in the early Universe and provide constraints on how the field was created and then amplified.

\subsection{SKA specifications}

The main specifications of the SKA design to allow the investigation of magnetic fields are:

- a frequency range $0.5-10 \mathrm{GHz}$, to give reliable information on the RM of targeted sources, solving the ambiguity related to its computation;

- an angular resolution of $0.1^{\prime \prime}$ at $1.4 \mathrm{GHz}$ to allow the resolution of structures smaller than $1 \mathrm{kpc}$ at $\mathrm{z} \geq 1$;

- large field of view, $\gtrsim 1 \mathrm{deg}^{2}$ at $1 \mathrm{GHz}$, to obtain observations of large portions of the sky in reasonably short times;

- large bandwith, > $400 \mathrm{MHz}$ at $1.4 \mathrm{GHz}$, to obtain RM values with a single frequency observations (RM survey);

- polarization purity of $-40 \mathrm{~dB}$ at the field center ( $-30 \mathrm{~dB}$ at the field edge) to allow measurement of the polarization parameters for sub-mJy sources;

- significant concentration $(>50 \%)$ of antennae in the central core $(\sim 5 \mathrm{~km})$, with minimum baseline of $20 \mathrm{~m}$, to allow the mapping of large scale structures, with high surface brightness sensitivity.

\section{Conclusions}

It is well established, from the observation of diffuse radio emission in clusters and from RM measurements of cluster and backgound radio sources, that the intracluster medium is permeated by large-scale magnetic fields of average intensity around $\sim \mu \mathrm{G}$. Several questions are still unanswered about the detailed structure of this field, its extent, its link to the cluster parameters, and its origin and evolution.

The presence of magnetic fields beyond clusters, in very rarified regions on the intergalactic medium, is inferred from the detection of low level synchrotron emission in a few cases. However, the information in this area is very scarce, because of the limitations of current instruments. Similarly, the data on high redshift objects, and generally on the high redhift Universe, are presently rather poor and unconclusive.

The observations with the SKA will provide the answer to many long-standing questions on magnetic fields. It will be possible to perform accurate experimental determination of large-scale 
magnetic fields in the intracluster medium, in the intergalactic space and in systems at high redshift. With the new data, it will be possible to map the evolution of magnetic fields from the early Universe to the present epoch, and distinguish between different models of magnetic field origin, seed field formation, and field amplification.

\section{Acknowledgments}

I am grateful to the organizers for the invitation to this interesting and fruitful school.

\section{References}

[1] S.W. Allen, G.B. Taylor, P.E.J. Nulsen, et al.: 2001, MNRAS 324, 842

[2] J. Bagchi, T. A. Enßlin, F. Miniati, C. S. Stalin, M. Singh, S. Raychaudhury, and N. B. Humeshkar: 2002, New Astronomy 7, 249

[3] J. Bagchi, F. Durret, G.B. Lima Neto, P. Surajit: 2006, Science 314, 791

[4] B. Ballarati, L. Feretti, A. Ficarra, G. Gavazzi, G. Giovannini, M. Nanni, M.C. Olori: 1981, A\&A 100,323

[5] J. D. Barrow, P.G. Ferreira, J. Silk: 1997, Phys. Rev. Let. 78, 3610

[6] R. Beck, B.M. Gaensler: 2004, NewAR 48, 1289

[7] P. Blasi, S. Burles, A.V. Olinto: 1999, ApJ 514, L79

[8] M.A. Brentjens, A.G. de Bruyn: 2005, A\&A 441, 1217

[9] G. Brunetti, G. Setti, L. Feretti, G. Giovannini: 2001, MNRAS 320, 365

[10] B.D.G. Chandran, S.C. Cowley, M. Ivanushkina, R. Sydora: 1999, ApJ 525, 638

[11] T.E. Clarke, P.P. Kronberg, H. Böhringer: 2001, ApJ 547, L111

[12] T.E. Clarke: 2004, Jour. Kor. Astr. Soc. 37, 337

[13] T.E. Clarke, T.A. Enßlin: 2006, AJ 131, 2900

[14] D.S. De Young: 1992, ApJ 386, 464

[15] K. Dolag, M. Bartelmann, H. Lesch: 1999, A\&A 348, 351

[16] K. Dolag, S. Schindler, F. Govoni, L. Feretti: 2001, A\&A 378, 777

[17] J. Eilek: 1999, in: Diffuse thermal and relativistic plasma in galaxy clusters, Eds. H. Böhringer, L. Feretti \& P. Schuecker, MPE Report 271, p. 71

[18] T. A. Enßlin, C. Vogt: 2003, A\&A 401, 835

[19] L. Feretti, D. Dallacasa, G. Giovannini, A. Tagliani: 1995, A\&A 302, 680

[20] L. Feretti, D. Dallacasa, F. Govoni, G. Giovannini, G.B. Taylor, U. Klein: 1999, A\&A 344, 472

[21] L. Feretti, M. Johnston-Hollitt: 2004, NewAR 48, 1145

[22] L. Feretti, C. Burigana, T.A. Enßlin: 2004, NewAR 48, 1137

[23] L. Feretti: 2005, AdSpR 36, 729 
[24] L. Feretti, G. Giovannini: 2007, in: Panchromatic View of Clusters of Galaxies and the Large-Scale Structure, Eds. M. Plionis, O. Lopez-Cruz, \& D. Hughes, in press, arXiv:astro-ph/0703494

[25] B.M. Gaensler: 2006, AN 327, 387

[26] B.M. Gaensler, R. Beck, L. Feretti: 2004, NewAR 48, 1003

[27] G. Giovannini, L. Feretti, T. Venturi, K.-T. Kim, P.P. Kronberg: 1993, ApJ 406, 399

[28] G. Giovannini, L. Feretti: 2004, Jour. Kor. Astr. Soc. 37, 323

[29] F. Govoni, L. Feretti, G. Giovannini, H. Böhringer, T.H. Reiprich, M. Murgia: 2001, A\&A 376, 803

[30] F. Govoni, G.B. Taylor, D. Dallacasa, L. Feretti, G. Giovannini: 2001, A\&A 379, 807

[31] F. Govoni, L. Feretti: 2004, Int. J. Mod. Phys. D 13 (N. 8), 1549

[32] F. Govoni, M. Murgia, L. Feretti, G. Giovannini, D. Dallacasa, G.B. Taylor: 2005, A\&A 430, L5

[33] F. Govoni, M. Murgia, L. Feretti, G. Giovannini, K. Dolag, G.B. Taylor: 2006, A\&A 460, 425

[34] D. Grasso, H.R. Rubinstein: 2001, Physics Report 348, 163

[35] U. Keshet, E. Waxman, A. Loeb: 2004, ApJ 617, 281

[36] K. T. Kim, P. P. Kronberg, G. Giovannini, and T. Venturi: 1989, Nature 341, 720

[37] T. Kolatt: 1998, ApJ 495, 564

[38] P.P. Kronberg, H. Lesch, U. Hopp: 1999, ApJ 511, 56

[39] P.P. Kronberg: 2006, AN 327, 517

[40] M Murgia, F Govoni, L. Feretti, G. Giovannini, D. Dallacasa, R. Fanti, G. B. Taylor, K. Dolag: 2004, A\&A 424, 429

[41] E. Orrú, M. Murgia, L. Feretti, F. Govoni, G. Brunetti, G. Giovannini, M. Girardi, G. Setti, G.: 2007, A\&A 467, 943

[42] M.J. Rees: 2006, AN 327, 395

[43] Y. Rephaeli: 1988, Comm. Mod. Phys. 12 (part C), 265

[44] K. Roettiger, J.M. Stone, J.O. Burns: 1999, ApJ 518, 594

[45] K. Subramanian, A. Shukurov, N.E.L. Haugen: 2006, MNRAS 366, 1437

[46] G.B. Taylor, R.A. Perley: 1993, ApJ 416, 554

[47] G.B. Taylor, F. Govoni, S.A. Allen, A.C. Fabian: 2001, MNRAS 326, 2

[48] C. Vogt, T. A. Enßlin: 2003, A\&A 412, 373

[49] H. J. Völk, A. M. Atoyan: 1999, Astrop. Phys. 11, 73

[50] M.A.G. Willson: 1970, MNRAS 151, 1 